\title{
NOTE ON LOCAL STRUCTURE OF ARTIN STACKS
}

\author{
ISAMU IWANARI
}

Let $\mathcal{X}$ be an Artin stack over $\mathbb{Z}$ (see [8]). A coarse moduli map (space) for $\mathcal{X}$ is a morphism $\pi: \mathcal{X} \rightarrow X$ to an algebraic space $X$ which has properties:

- $\pi$ is universal among morphisms from $\mathcal{X}$ to algebraic spaces, i.e., for any morphism $\phi: \mathcal{X} \rightarrow Y$ with an algebraic space $Y$ there is a unique morphism $f: X \rightarrow Y$ such that $\phi=f \circ \pi$,

- for any algebraically closed field $K, \pi$ identifies the set of isomorphism classes of $\mathcal{X}(K)$ with the set $X(K)$ of $K$-valued points of $X$.

Let $\mathcal{X}$ be an Artin stack locally of finite type over a locally noetherian scheme $S$. Suppose that $\mathcal{X}$ has finite inertia stack, that is, the projection

$$
\operatorname{pr}_{1}: I_{\mathcal{X}}:=\mathcal{X} \times_{\mathcal{X}_{\times_{S}} \mathcal{X}} \mathcal{X} \longrightarrow \mathcal{X}
$$

is finite. In this setting, according to [6] there exist an algebraic space $X$ locally of finigte type over $S$ and a coarse moduli map $\pi: \mathcal{X} \rightarrow X$ over $S$, where $\pi$ is proper and quasi-finite. When in addition $\mathcal{X}$ is a Deligne-Mumford stack, it has, étale locally on its coarse moduli space $X$, the form of a quotient stack $[Z / G]$ where $Z$ is an affine scheme and $G$ is a finite group. This structure has been important and very useful in various situations. We would like to make the following useful observation:

Theorem 1. Let $\mathcal{X}$ be an Artin stack locally of finite type over $S$ with finite inertia stack. Then for any point $x \in \mathcal{X}$ there is an étale neighborhood $U \rightarrow X$ of $\pi(x)$ such that the $S$-stack $\mathcal{X} \times{ }_{X} U$ has the form $\left[\operatorname{Spec} R / \mathrm{GL}_{n}\right]$, where $\operatorname{Spec} R$ is an affine scheme over $S$ and $\mathrm{GL}_{n}$ is the general linear group scheme defined over $S$. Moreover, $U$ is naturally isomorphic to the spectrum $\mathrm{Spec} R^{\mathrm{GL}_{n}}$ of the invariant ring.

Proof. Let $\pi(x)=y$. Then according to the proof of the theorem of Keel and Mori (see [6] in particular Section 4 in loc. cit.), we see that there is an étale neighborhood $U \rightarrow X$ of $y$ from an affine scheme $U$ such that $\mathcal{X}_{U}:=\mathcal{X} \times_{X} U$ has a finite flat surjective morphism $p: Y \rightarrow \mathcal{X}_{U}$ from a scheme $Y$. Then since $Y$ is a scheme, $p_{*} \mathcal{O}_{Y}$ is a vector bundle on $\mathcal{X}_{U}$ such that at each geometric point the stabilizer faithfully acts on the fiber. After shrinking $\mathcal{X}_{U}$ if necessary we assume that $\mathcal{X}_{U}$ is connected. Then by $[3,2.12]$ the total space of the corresponding principal $\mathrm{GL}_{n}$-bundle $W$ over $\mathcal{X}_{U}$ is an algebraic space and thus $\mathcal{X}_{U}$ is isomorphic to the quotient stack $\left[W / \mathrm{GL}_{n}\right]$ where $n$ is a non-negative integer. Now we will show that $W$ is an affine scheme. Note that the projection $W \rightarrow\left[W / \mathrm{GL}_{n}\right]$ is a $\mathrm{GL}_{n}$-bundle and $\mathcal{X}_{U} \simeq\left[W / \mathrm{GL}_{n}\right] \rightarrow U$ is proper. Hence $W$ is separated and noetherian. In addition, $Y \rightarrow \mathcal{X}_{U} \rightarrow U$ is a finite morphism and thus $Y$ is affine. Therefore $W \times \mathcal{X}_{U} Y$ is affine because the second projection $W \times \mathcal{X}_{U} Y \rightarrow Y$ is affine. Notice that the first projection $W \times_{\mathcal{X}_{U}} Y \rightarrow W$ is a finite surjective morphism. Consequently, applying Chevalley's theorem for algebraic

The author is supported by JSPS. 
spaces (see [7, III 4.1]) we deduce that $W$ is affine. The last claim follows from the fact that $\mathcal{X}_{U} \rightarrow U$ is a coarse moduli map because $U \rightarrow X$ is étale.

Remark 2. Suppose further that $\mathcal{X}$ is normal in Theorem 1 . Then we can take an action of $\mathrm{GL}_{n}$ on Spec $R$ in Theorem 1 to be linearized. Note that under the assumption $\operatorname{Spec} R$ is normal. According to [13, Theorem 3.3, 2.18] (see also [10, 2.5]), there is a $\mathrm{GL}_{n}$-vector bundle $\mathcal{V}$ on $U$ and a $\mathrm{GL}_{n}$-equivariant immersion $\operatorname{Spec} R \rightarrow \mathbb{P}(\mathcal{V})$.

Remark 3. If $\mathcal{X}$ is a Deligne-Mumford stack of finite type over $S$ which has finite inertia stack, then étale locally on its coarse moduli space, $\mathcal{X}$ is the quotient $[Y / G]$ of an affine scheme $Y$ by an action of a finite (constant) group $G$ (see [1]). If stabilizer group schemes at geometric points on $\mathcal{X}$ are finite (not necessarily reduced) linearly reductive group schemes, in [2] it is shown that $\mathcal{X}$ is, étale locally on its coarse moduli space, the quotient of an affine scheme by an action of a linearly reductive group scheme. In [4] such étale-local quotient structures was studied when $\mathcal{X}$ has (not necessarily finite) linearly reductive stabilizers and satisfies the stability (see [4]). In these cases the stabilizer group schemes has no non-trivial deformation (i.e., has a unique deformation), and we may take $G$ to be the stabilizer group scheme at a point on $\mathcal{X}$ when we work over a field. This point is crucial for Luna's étale slice theorem. However, in positive characteristic case a general (finite) group scheme has many and rich deformations. Thus in general local structures of Artin stacks are not so simple as above cases. Indeed an Artin stack can contain the information arising from non-trivial flat deformations of $G$, i.e., $B G$.

The typical usage of Theorem 1 is the reduction of problems to the case of group actions. In the rest of this note, we will present one of such applications of Theorem 1, which is a direct one. For this we shall prepare our setup.

Let $\mathcal{X}$ be an Artin stack of finite type over $\mathbb{Z}$. We will denote by $G(\mathcal{X})(\operatorname{resp} . K(\mathcal{X}))$ the algebraic K-theory spectrum of the exact category of coherent sheaves (resp. vector bundles) on $\mathcal{X}$, and we let $G(\mathcal{X}) \otimes \mathbb{Q}$ and $K(\mathcal{X}) \otimes \mathbb{Q}$ Bousfield localizations of $G(\mathcal{X})$ and $K(\mathcal{X})$ respectively, with respect to $\mathbb{Q}$ (see [5]). Let us recall the isovariant étale descent of $G$-theory due to Joshua [5], which generalizes Thomason's descent [11]. A morphism $\mathcal{Y} \rightarrow \mathcal{X}$ of Artin stacks is isovariant if $I_{\mathcal{Y}} \rightarrow I_{\mathcal{X}} \times \mathcal{X} \mathcal{Y}$ is an isomorphism, where $I_{\mathcal{X}}$ and $I_{\mathcal{Y}}$ denote inertia stacks of $\mathcal{X}$ and $\mathcal{Y}$ respectively. Note that an isovariant morphism is representable. If $\mathcal{X} \rightarrow Z$ is a morphism to an algebraic space $Z$ and $W \rightarrow Z$ is a morphism of algebraic spaces, then the projection $\mathcal{X} \times{ }_{Z} W \rightarrow \mathcal{X}$ is isovariant. For any Artin stack $\mathcal{X}$ we will denote by $\mathcal{X}_{\text {iso.et }}$ the site whose objects are isovariant étale morphisms $\mathcal{Y} \rightarrow \mathcal{X}$. A morphism from $y: \mathcal{Y} \rightarrow \mathcal{X}$ to $y^{\prime}: \mathcal{Y}^{\prime} \rightarrow \mathcal{X}$ in $\mathcal{X}_{\text {iso.et }}$ is a pair $\left(f: \mathcal{Y} \rightarrow \mathcal{Y}^{\prime}, \sigma\right)$ where $\sigma: y \simeq y^{\prime} \circ f$. Then using Quillen's Q-construction and the loop functor, we have two presheaves of spectra

$$
\mathbf{G}:\left(\mathcal{X}_{\text {iso.et }}\right)^{o p} \longrightarrow \mathbf{S p t}
$$

and

$$
\mathbf{K}:\left(\mathcal{X}_{\text {iso.et }}\right)^{o p} \longrightarrow \mathbf{S p t}
$$

which to any $\mathcal{Y} \rightarrow \mathcal{X}$ in $\mathcal{X}_{\text {iso.et }}$ associate $G(\mathcal{Y})$ and $K(\mathcal{Y})$ respectively, where Spt is the category of spectra. For a presheaf of spectra $P$ let $\mathbb{H}_{\text {iso.et }}(\mathcal{X}, P)$ be the hypercohomology with respect to isovariant étale topology on $\mathcal{X}$, defined in [5, 4.2.3]. In loc. cit., to define hypercohomology of presheaves of spectra the author uses Godement 
resolutions. If you are familiar with model categories, you may consider the hypercohomology of $P$ to be $P^{\prime}(\mathcal{X})$ where $P \rightarrow P^{\prime}$ is a fibrant replacement in the category of presheaves of spectra endowed with the Jardine's model structure $[9,3.3]$ with respect to isovariant étale topology. Similarly, for an algebraic space $X$ and any presheaf of spectra $P$ on the étale site on $X$, we write $\mathbb{H}_{e t}(X, P)$ for the hypercohomology of $P$ with respect to étale topology. The descent theorem $[5,5.10]$ says that there is a weak equivalence $G(\mathcal{X}) \otimes \mathbb{Q} \rightarrow \mathbb{H}_{\text {iso.et }}(\mathcal{X}, \mathbf{G} \otimes \mathbb{Q})$. (See [5] for various localized versions.) The following generalizes Poincaré duality [5, 5.16], which was proved in the case of Deligne-Mumford stacks.

Proposition 4 (Poincaré duality). Let $\mathcal{X}$ be a regular Artin stack of finite type over $\mathbb{Z}$ with finite inertia stack. Let $\pi: \mathcal{X} \rightarrow X$ be a coarse moduli map and $\pi_{\#}$ the direct image functor of presheaves of spectra. Then the natural map

$$
G(\mathcal{X}) \otimes \mathbb{Q} \simeq \mathbb{H}_{e t}\left(X, \pi_{\#} \mathbf{G} \otimes \mathbb{Q}\right) \leftarrow \mathbb{H}_{e t}\left(X, \pi_{\#} \mathbf{K} \otimes \mathbb{Q}\right)
$$

is a weak equivalence of spectra.

Proof. To show our claim, clearly we may work étale locally on the coarse moduli space $X$. Thus according to Proposition 1 we may and will assume that $\mathcal{X}$ is of the form $\left[\operatorname{Spec} R / \mathrm{GL}_{n}\right]$. Now we can apply the result of Thomason [12, Theorem 5.7] to obtain our Proposition.

Remark 5. Proposition 4 also holds for other localized $G$-theories [5, 5.1.5].

\section{REFERENCES}

[1] D. Abramovich and A. Vistoli, Compactifying the space of stable maps, Jour. AMS., 15 (2005) $27-75$.

[2] D. Abramovich, M. Olsson, and A. Vistoli, Tame stacks in positive characteristic, Ann. Inst. Fourier (Grenoble) 58 (2008), 1057-1091.

[3] D. Edidin, B. Hassett, A. Kresch, and A. Vistoli, Brauer groups and quotient stacks, Amer. J. Math. 123 (2001), 761-777.

[4] I. Iwanari, Stable points on algebraic stacks, Advances in Math. (2010). 257-299.

[5] R. Joshua, Riemann-Roch for algebraic stacks:I, Compositio Math., 136 (2003), 117-169.

[6] S. Keel and S. Mori, Quotients by groupoids, Ann. of Math, 145 (1997), 193-213.

[7] D. Knutson, Algebraic spaces, Lecture Notes in Math. 203 Springer (1971)

[8] G. Laumon and L. Moret-Bailly, Champs Algébriques, Springer-Verlag (2000).

[9] S. Mitchell, Hypercohomology spectra and Thomason's descent theorem, preprint.

[10] H. Sumihiro, Equivariant completion II, J. Math. Kyoto Univ. 15, (1975) 573-605.

[11] R. W. Thomason, Algebraic K-theory and étale cohomology, Ann. Sci. Ec. Norm. Sup. 18 (1985), $437-552$.

[12] R. W. Thomason, Algebraic K-theory of group scheme actions, In: W. Browder (ed.) Algebraic topology and Algebraic K-theory (Ann. Math. Stud. 113, pp. 539-563) Princeton, NJ:Princeton Univ. 1987.

[13] R. W. Thomason, Equivariant resolution, Linearization, and Hirbert's Fourteenth Problem over Arbitarary Base schemes, Advances in Math. 65, (1987) 16-34.

Research Institute of the Mathematical Sciences, Kyoto University, Kyoto, 6068502, JAPAN

E-mail address: iwanari@kurims.kyoto-u.ac.jp 